\title{
Late Results of Anterior Cervical Discectomy and Fusion with Interbody Cages
}

\author{
Murat Dağl ${ }^{1}$, Uygur Er ${ }^{2}$, Serkan Şimşek ${ }^{1}$, Murad Bavbek $^{1}$ \\ ${ }^{I}$ Neurosurgery Clinic, Dışkapı Yıldırım Beyazıt Education and Research Hospital, Ankara, Turkey \\ ${ }^{2}$ Neurosurgery Clinic, Koru Hospital, Ankara, Turkey
}

\begin{abstract}
Study Design: Retrospective analysis.
Purpose: To evaluate the effectiveness of anterior cervical discectomy with fusion for degenerative cervical disc disease.

Overview of Literature: Anterior spinal surgery originated in the mid-1950s and graft for fusion was also employed. Currently anterior cervical microdiscectomy and fusion with an intervertebral cage is a widely accepted procedure for treatment of cervical disc hernia. Artificial grafts and cages for fusion are preferred because of their lower morbidity, reduced operating time and acceptable fusion rate.

Methods: The study involved retrospective analysis and investigation of long-term results for 41 consecutive patients who had undergone anterior cervical discectomy and fusion with an intervertebral cage for cervical disc hernia. The angle of lordosis, segmental height and range of motion were evaluated preoperatively and postoperatively at 1 month and 2 years. The clinical outcome was assessed by the visual analog scale and Odom's criteria.

Results: The angle of lordosis increased by $2.62^{\circ}$ and the range of motion angle increased by $5.14^{\circ}$ after the operation. The segmental height did not change. The visual analog scale and Odom's criteria scores decreased significantly after the operation.

Conclusions: Using a cage in anterior cervical discectomy prevents segmental collapse, so the segmental height and the angle of lordosis are preserved and newly-developed pain does not occur.
\end{abstract}

Keywords: Cervical vertebral fusion; Cervical lordosis; Outcome assesment; Range of motion; Polyetheretherketone cage

\section{Introduction}

Until the 1950s, cervical spine surgery was largely approached posteriorly [1]. In the mid-1950s, pioneers such as Smith and Robinson, Cloward, and Bailey and Badgley initiated the notion of anterior spine surgery [1,2]. At that time Smith and Robinson, and also Cloward used a graft for fusion [1]. Currently anterior cervical microdiscectomy (ACD) and fusion (ACDF) with an intervertebral cage is a widely accepted procedure for cervical disc hernia treatment
$(\mathrm{CDH})$. Artificial grafts and cages for fusion are preferred nowadays because of their lower morbidity, reduced operating time and acceptable fusion rate [1,3-5]. An anterior plate system can be added to the procedure which can prevent cage subsidence and may contribute to the developing fusion by compressing interbody space. Using a bladed cage may provide sufficient resistance to subsidence. The contribution of the fusion rate of an anterior plate is only $4 \%$ to $5 \%$ [6]. To avoid complications related to an anterior plate system, bladed cages were preferred to adding an anterior plate in

Received Mar 14, 2012; Revised Apr 12, 2012; Accepted May 9, 2012

Corresponding author: Uygur Er

Neurosurgery Clinic, Koru Hospital, Söğütözü C., 4th Sk, No: 22/7, 06510, Ankara, Turkey

Tel: +90-505-589-23-55, Fax: +90-312-284-51-02, E-mail: uygurer@gmail.com 
this study. Long-term results of ACDF are presented in this study.

\section{Materials and Methods}

The study involved retrospective analysis and investigation of long-term results for 41 consecutive patients on whom ACDF for CDH were performed between 2005 and 2008. After Institutional Review Board approval (09.06.2019/0109), written informed consent was obtained from every patient. Institutional Review Board approval was obtained and each patient provided informed consent.

Criteria for inclusion were as follows: 1) Patients between the ages of 18 and 70 with CDH. 2) Patients with pain that had not responded to medical therapy for at least 3 weeks. 3) Symptomatically and radiologically coherent patients.4) Patients with soft disc hernia.

Exclusion criteria were: 1) Patients with cervical spondylosis, and spondylotic myelopathy. 2) Patients with hard disc or foraminal spurs. 3) Patients with cervical trauma. 4) Patients who had had previous cervical surgery for any reason. 5) Patients who had received radiotherapy or chemotherapy. 6) Pregnant patients. 7) Patients who did not attend regularly for follow-up examinations.

Consider listing in paragraph format. Example: exlusion criteria were patients with: 1) cervical spondylosis, and spondylotic myelopathy, 2) hard disc or foraminal spurs...

\section{Demographic data and clinical outcomes}

The mean age of the patients was 47.07 years (range, 30 to 70 years). The study included 22 (53.7\%) female and 19 (46.3\%) male patients. The mean duration of symptoms was 6 months (between 2 weeks and 16 months). Radiculopathy was the leading symptom.

All patients received anterior cervical microdiscectomy. Operations were performed under intratracheal general anesthesia in a supine position with the head slightly extended. After the platysma was cut, dissection progressed bluntly to the anterior aspects of the cervical vertebrae. After the fluoroscopic vertebral level control, the entire cervical disc was excised by micro technique under the operating microscope. The posterior longitudinal ligament was opened and the dura was seen. Both endplates were scratched out by curettes. After measurement of the height and depth of the intervertebral space, an appropriate lordotic polyether ether ketone cage (Norm Medical Products, Ankara, Turkey) was inserted into the intervertebral space with $1 \mathrm{~mL}$ demineralized bone matrix (Tissuenet, Orlando, FL, USA) inside it. Cages were lordotic and bladed, and dimensions of the cages were decided after measurement of the intervertebral spaces of each individual patient. After the last fluoroscopic control for cage dimensions and localization, hemostazis was performed and the surgical closure was completed.

\section{Outcome measures and radiographic assessments}

Antero-posterior and lateral cervical roentgenography, cervical spinal magnetic resonance imaging, cervical spinal and bony window computed tomography (CT) were performed on all patients preoperatively. Patients were assessed postoperatively at 1 month and at 2 years. Angle of lordosis (AL), range of motion (ROM) angle and segmental height (SH) of the patients were calculated preoperatively and postoperatively at 1 month and at 2 years. SH was measured at the level where ACD would be performed. Myelopathic changes and adjacent segment disease were evaluated after 2 years. A visual analog scale (VAS) and Odom's criteria were used for the evaluation of patients clinically. AL is defined as the angle between the line parallel to the $\mathrm{C} 2$ posterior margin and the line parallel to the $\mathrm{C} 7$ posterior margin on the lateral cervical roentgenogram [7]. If this angle is between $10^{\circ}$ and $40^{\circ}, \mathrm{AL}$ is accepted as normal, if the angle is between $0^{\circ}$ and $10^{\circ}, \mathrm{AL}$ shows lessen in the lordosis, and if $\mathrm{AL}<0^{\circ}$, the cervical vertebrae are considered kyphotic [8]. ROM angle is defined as an angle between the line parallel to the superior end plate of $\mathrm{C} 2$ and the line parallel to the superior endplate of C7. A mean angle of $20^{\circ}$ is considered normal $[8,9]$.

One-level ACDF was performed on 29 of the patients and two-level ACDF was performed on 12 patients.

\section{Statistical analysis}

SPSS ver. 15.0 for windows (SPSS Inc., Chicago, IL, USA) was used for statistical analysis. Frequency tables for categorical variables and descriptive statistics (mean, median, standard deviation, minimum-maximum values) for numerical variables were given. Statistical significance of categorical variables between groups was determined by chi-square. Numerical comparisons in data with normal distribution were carried out by T-test for independent variables and analysis of variance for dependent variables, by Mann Whitney $U$ Test for independent variables and by Friedman Test for dependent variables for data without normal distribu- 
tion. The results were taken as statistically significant if the p-value was less than 0.05 .

\section{Results}

\section{Radiographic assessments}

Both of the AL and ROM angle measurements after the first month and at two years after the operation showed a significant increase $(p<0.001)$, shown in Figs. 1,2 . There was no significant change in the $\mathrm{SH}$ values shown in Fig. 3.

\section{Clinical outcome}

Both of the VAS and Odom's criteria scores decreased significantly in the first month and at two years after the operation $(p<0.001)$, shown in Figs. $4,5$.

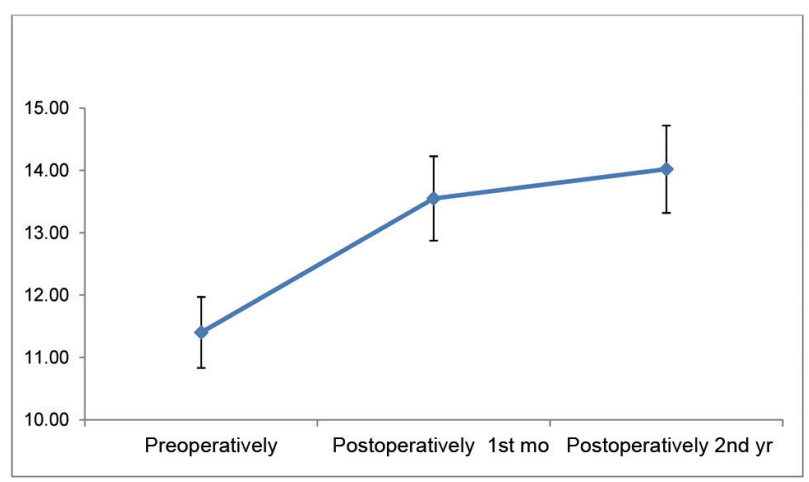

Fig. 1. Changes in angle of lordosis.

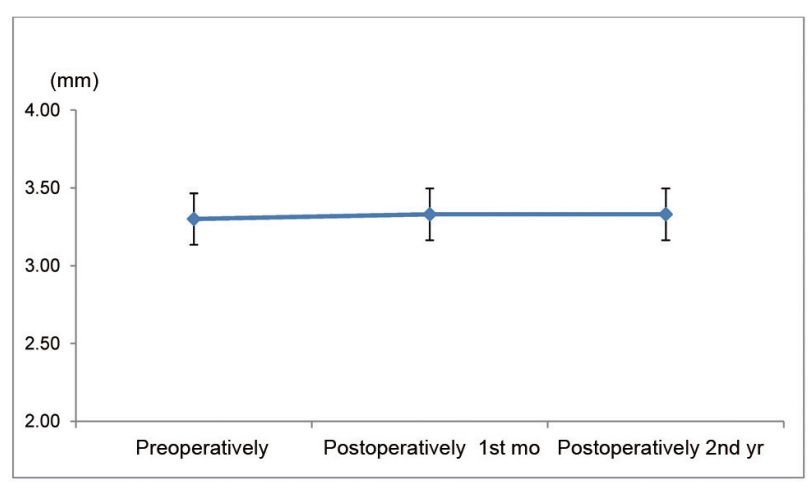

Fig. 3. Changes in segmental height.

\section{Complications}

There was no occurrence of vascular damage, dural tears, tracheal and esophageal injuries in the operations. No hematoma was found in this series. Temporary hoarseness was found in two patients. Cage subsidence was not seen in the follow-up period. Adjacent segment degenerations were seen in nine patients. None of the patients were operated on for degenerative diseases. Wound infection was seen in one patient, and was treated with antibiotics.

\section{Discussion}

Soft CDHs cause symptoms by compressing the spinal cord anteriorly or anterolaterally. If there is a surgical indication for treatment of a $\mathrm{CDH}$, decompression of the spinal cord by ACD relieves the symptoms. The aim of all surgical procedures is to decompress nerve roots and the spinal cord and alleviate pain. However, segmental collapse, caused

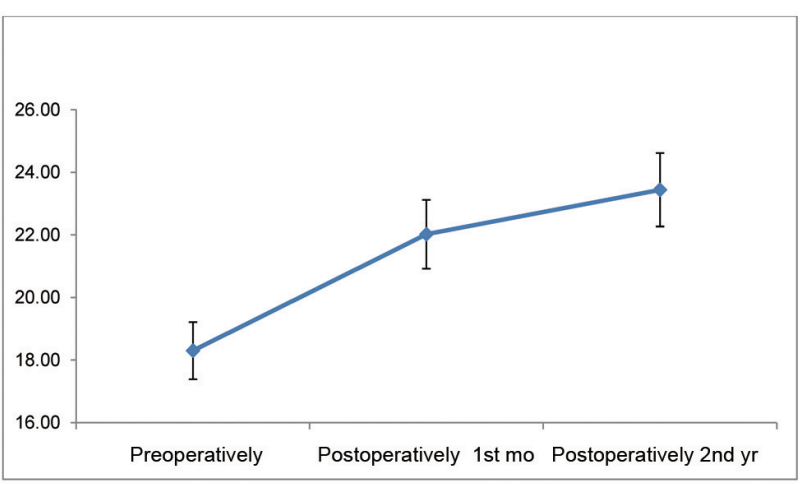

Fig. 2. Changes in range of motion.

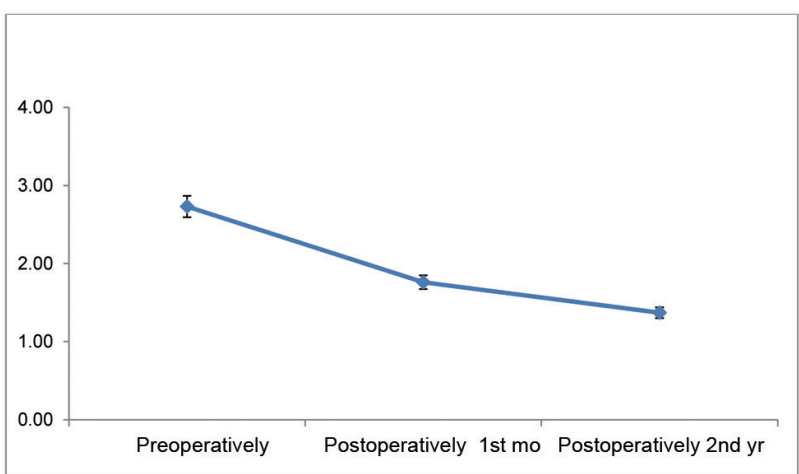

Fig. 4. Changes in Odom's criteria. 
by losing $\mathrm{SH}$ because of the removal of disc material, and consequently changing of $\mathrm{AL}$, become new sources of pain and discomfort for the patient [10]. After a simple ACD procedure the cervical foraminal area diminishes and new symptoms of cervical root compression can be evident [11]. Besides, removing disc material entirely results in instability in the cervical spine because of lack of support to the anterior column. The need to preserve $\mathrm{SH}$ and restore $\mathrm{AL}$ after an $\mathrm{ACD}$, and the fact that supporting the anterior column can prevent symptoms depending on these changes, led to the idea of including fusion in ACD operations. However, some distraction may occur after operation and the gap can be reduced to some extent. SH seemed unchanged after the procedures in this study. This situation may arise from that thickness of interbody cages which were used after measuring the thickness of interbody spaces. Distraction was avoided by using just the right size interbody cage instead of a cage that distracts the interbody space.

Some diminishment of ROM was expected after the fusion. In this study the ROM angle was measured instead of just the ROM. ROM angle is a different notion that it is defined as an angle between the line parallel to the superior end plate of $\mathrm{C} 2$ and the line parallel to the superior endplate of C7 $[8,9]$. As a result the ROM angle may increase after operation by increasing lordosis. In this study, little increase in the ROM angles was detected. This may be because of improvement in pain. This change may not be considered structural.

There are many methods for providing fusion. Autograft, allograft, cage application and anterior plating are widely used methods. No statistically significant difference has been found between these four major methods [4]. The graft used for fusion can easily move after the operation unless

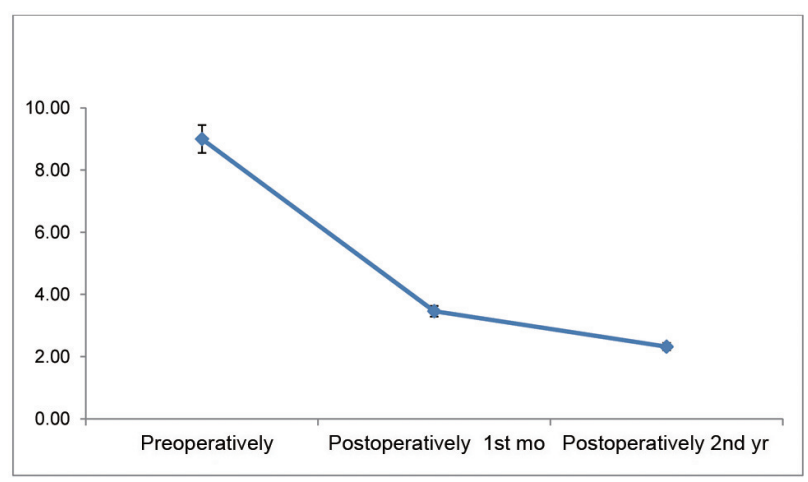

Fig. 5. Changes in visual analog scale. stabilization is performed. Furthermore, graft materials can be compressed, so $\mathrm{SH}$ and AL cannot be achieved. In addition to all of these, graft particles can move to epidural areas, leading to new compression sites. Fusion materials were put in a cage to avoid graft movements and compression. At the same time, cage applications preserve $\mathrm{SH}$ and $\mathrm{AL}$ within normal limits. Slipping of grafts that have a cage was still observed but less frequently. An anterior plate can prevent anterior slipping and can also compress the graft to fuse quickly. Developing technology produced cages that hold on to the vertebral end plates better. These cages minimized the need for an anterior plate.

All of these developments have made ACDF a popular surgical option for treatment of soft $\mathrm{CDH}$ [11]. It provides a wider angle of sight, enables removal all of the disc material and osteofits, makes possible bony decompression and sufficient fusion, and makes ACDF the preferred technique for treatment of soft CDH $[2,10,12,13]$.

Adding fusion to the ACD operation diminishes $\mathrm{SH}$ losses and in parallel prevents foraminal compressions [14]. Although autografts provide better fusion rates, because of donor site complications and the fact that it requires more time, cage and artificial grafts are preferred [5,15]. Prevention of postoperative kyphosis is another advantage of cage fusion. Lordotic cages in particular can provide normal cervical alignment [16]. This technique can also put $\mathrm{AL}$ and ROM within normal limits, so postoperative pain reduces and quality of life of the patients increases $[9,17]$. Changes in Odom's criteria and VAS show that clinical improvement is parallel to these radiological measurements. In particular a sharp decline in VAS after one month of the operation may be considered as evidence of the effectiveness of this procedure.

Subsidence of the cage is another issue that needs to be considered. Many reports in the literature describe risk factors for cage subsidence $[11,18]$. Using a bladed cage may eliminate this problem to a reasonable degree. The blades of the cage hang on the vertebral end plates well, and subsidence of the cage can be prevented. In this study, subsidence of the cage was not encountered, because bladed cages were used instead of regular cages. The small population of this study may be a factor for the absence of subsidence.

\section{Conclusions}

ACDF is an effective way for treatment of $\mathrm{CDH}$. Using a cage prevents segmental collapse, so $\mathrm{SH}$ and $\mathrm{AL}$ are saved 
and do not result in newly developed pain. Using a bladed cage instead of regular cage may prevent cage subsidence. The fusion rate was not taken into consideration in this study, but it should, because it is one of the important limitations of this study. Future studies that care about the fusion rate will bring clarity.

\section{Conflict of interest}

No potential conflict of interest relevant to this article was reported.

\section{References}

1. Cloward RB. The anterior approach for removal of ruptured cervical disks. J Neurosurg 1958;15:602-17.

2. Fielding JW. Cervical spine surgery. Past, present, and future potential. Clin Orthop Relat Res 1985;(200):28490.

3. Arts MP, Brand R, van den Akker E, Koes BW, Peul WC. The NEtherlands Cervical Kinematics (NECK) trial. Cost-effectiveness of anterior cervical discectomy with or without interbody fusion and arthroplasty in the treatment of cervical disc herniation; a double-blind randomised multicenter study. BMC Musculoskelet Disord 2010;11:122.

4. Bhadra AK, Raman AS, Casey AT, Crawford RJ. Singlelevel cervical radiculopathy: clinical outcome and costeffectiveness of four techniques of anterior cervical discectomy and fusion and disc arthroplasty. Eur Spine J 2009;18:232-7.

5. Lied B, Roenning PA, Sundseth J, Helseth E. Anterior cervical discectomy with fusion in patients with cervical disc degeneration: a prospective outcome study of 258 patients (181 fused with autologous bone graft and 77 fused with a PEEK cage). BMC Surg 2010;10:10.

6. Fraser JF, Hartl R. Anterior approaches to fusion of the cervical spine: a metaanalysis of fusion rates. J Neurosurg Spine 2007;6:298-303.

7. Penning L. Normal movements of the cervical spine. AJR Am J Roentgenol 1978;130:317-26.
8. Ara T, Iizuka H, Sorimachi Y, et al. Evaluation of neck pain by using a visual analog scale before and after laminoplasty in patients with cervical myelopathy: relationship with clinical results. J Neurosurg Spine 2010;12:635-40.

9. Kim SW, Limson MA, Kim SB, et al. Comparison of radiographic changes after ACDF versus Bryan disc arthroplasty in single and bi-level cases. Eur Spine J 2009;18:218-31.

10. Watters WC 3rd, Levinthal R. Anterior cervical discectomy with and without fusion: results, complications, and long-term follow-up. Spine (Phila Pa 1976) 1994;19:2343-7.

11. Ha SK, Park JY, Kim SH, Lim DJ, Kim SD, Lee SK. Radiologic Assessment of Subsidence in Stand-Alone Cervical Polyetheretherketone (PEEK) Cage. J Korean Neurosurg Soc 2008;44:370-4.

12. Caspar W, Barbier DD, Klara PM. Anterior cervical fusion and Caspar plate stabilization for cervical trauma. Neurosurgery 1989;25:491-502.

13. Connolly ES, Seymour RJ, Adams JE. Clinical evaluation of anterior cervical fusion for degenerative cervical disc disease. J Neurosurg 1965;23:431-7.

14. Topuz K, Colak A, Kaya S, et al. Two-level contiguous cervical disc disease treated with peek cages packed with demineralized bone matrix: results of 3-year follow-up. Eur Spine J 2009;18:238-43.

15. Chau AM, Mobbs RJ. Bone graft substitutes in anterior cervical discectomy and fusion. Eur Spine J 2009;18:44964.

16. Lange M, Philipp A, Fink U, Oeckler R. Anterior cervical spine fusion using RABEA-Titan-Cages avoiding iliac crest spongiosa: first experiences and results. Neurol Neurochir Pol 2000;34:64-9.

17. Bertalanffy H, Eggert HR. Complications of anterior cervical discectomy without fusion in 450 consecutive patients. Acta Neurochir (Wien) 1989;99:41-50.

18. Bartels RH, Donk RD, Feuth T. Subsidence of standalone cervical carbon fiber cages. Neurosurgery 2006;58:502-8. 